\title{
Patrones de aprendizaje del profesorado colombiano en formación
}

\author{
Evolution of learning patterns among Colombian \\ pre-service teachers
}

Christian Hederich-MartíneZ

Departamento de Psicopedagogía

Facultad de Educación

Universidad Pedagógica Nacional

Calle 72 No. 11-86, Bogotá (Colombia)

hederich@pedagogica.edu.co

https://orcid.org/0000-0003-1080-9973

\section{Angela Camargo-Uribe}

Departamento de Lenguas

Facultad de Humanidades

Universidad Pedagógica Nacional

Calle 72 No. 11-86, Bogotá (Colombia)

acamargo@pedagogica.edu.co

https://orcid.org/0000-0003-3611-810X

Resumen: A fin de comprender el cambio de los patrones de aprendizaje de maestros en formación a lo largo de la carrera, 1.016 estudiantes de una universidad pedagógica de Bogotá, Colombia, respondieron al Inventario de Estilos de Aprendizaje (ILS). La muestra estaba dividida según el momento de la carrera: inicio, intermedio y final. Se realizó un análisis de conglomerados sobre los factores obtenidos en un análisis de componentes principales. Los resultados indican cuatro patrones: uno orientado al significado y aplicación
Carolina HernándeZ-VALbUENA

Departamento de Psicopedagogía

Facultad de Educación

Universidad Pedagógica Nacional

Calle 72 No. 11-86, Bogotá (Colombia)

chernandezv@pedagogica.edu.co

https://orcid.org/000o-0003-3280-2975

del conocimiento, uno dirigido a su acumulación, otro no dirigido y un patrón "inactivo", con bajas respuestas en todas las escalas. La proporción de estudiantes por patrón cambia entre los primeros y últimos semestres, ganando progresivamente mayor prevalencia la orientación hacia el significado.

Palabras clave: Formación de profesores, Aprendizaje, Diferencias individuales, Patrones de aprendizaje. 
Abstract: To understand the change of learning patterns among pre-service teachers, 1016 students attending a pedagogical university in Bogotá, Colombia, answered the Inventory of Learning Styles (ILS). The sample was divided according to the moment of the career: at the beginning; in the middle and at the end. A cluster analysis was done on the factors obtained by a principal component analysis. Findings indicate four learning patterns: one directed towards meaning and application of knowledge, one directed toward knowledge accumulation, an undirected pattern, and an "inactive" pattern, with low responses in all scales. The proportion of students in each pattern changes throughout the career, with the meaning directed pattern progressively gaining more prevalence.

Keywords: Teacher training, Learning, Individual differences, Learning patterns.

\section{INTRODUCCIÓN}

A lcanzar sistemas educativos de calidad requiere formar profesores de excelencia. A fin de lograrlo, requerimos comprender de manera profunda las formas de aprendizaje de la población que se prepara para ser maestro y averiguar de qué manera la formación influye en estos procesos. Lo anterior se justifica en la medida en que, por una parte, las formas de aprender de los docentes son un factor que incide de manera directa en su forma de enseñar (Camargo-Uribe, 2015; Finson, Thomas y Pedersen, 2006; Saracho, 2003) y, por otra, ciertas formas de aprender son más deseables para el futuro docente y son ellas las que deberían enseñarse durante el proceso formativo (Vermunt, 2007; Vermunt y Verloop, 1999). Ello es especialmente importante en el caso de la formación de profesores, dadas sus implicaciones tanto para la futura labor docente como para las poblaciones estudiantiles con las que estos maestros ejercerán su profesión.

Respecto del problema de identificar las formas de aprender de los profesores en formación, proponemos aquí, siguiendo a Vermunt (1998), una mirada diferencial por medio del concepto de patrón de aprendizaje. En contraste con otros conceptos afines, como los de estilo cognitivo o estilo de aprendizaje, el patrón de aprendizaje es bastante maleable. No es un rasgo inherente al aprendiz, sino que es, más bien, el resultado de influencias personales y contextuales presentes en la experiencia, por lo que puede cambiar como consecuencia de ella (Vermunt y Donche, 2017; Vermunt y Endedijk, 2011). Así, el concepto de patrón resulta útil para estudiar la compleja relación entre el aprendizaje y la enseñanza en el futuro profesor.

Teniendo en cuenta lo anterior, los propósitos de este estudio son: 1) identificar los patrones de aprendizaje de docentes en formación, y 2) describir los cambios de estos patrones a lo largo de los estudios. 


\section{EL MODELO DE PATRONES DE APRENDIZAJE DE J. D. VERMUNT}

De acuerdo con Vermunt (2005), un patrón de aprendizaje corresponde a aquellas actividades, creencias y motivaciones relacionadas con el aprendizaje, que caracterizan a un estudiante en algún momento de su vida académica. El constructo intenta articular 4 elementos en un todo coherente y comprensivo: 1) los modelos mentales o creencias sobre el aprendizaje; 2) las orientaciones o motivaciones hacia el aprendizaje; 3) las estrategias que regulan el proceso y 4) las estrategias de procesamiento durante el mismo. La Figura 1 presenta el modelo completo y sus relaciones.

\section{Figura 1. Dimensiones del aprendizaje según Vermunt}

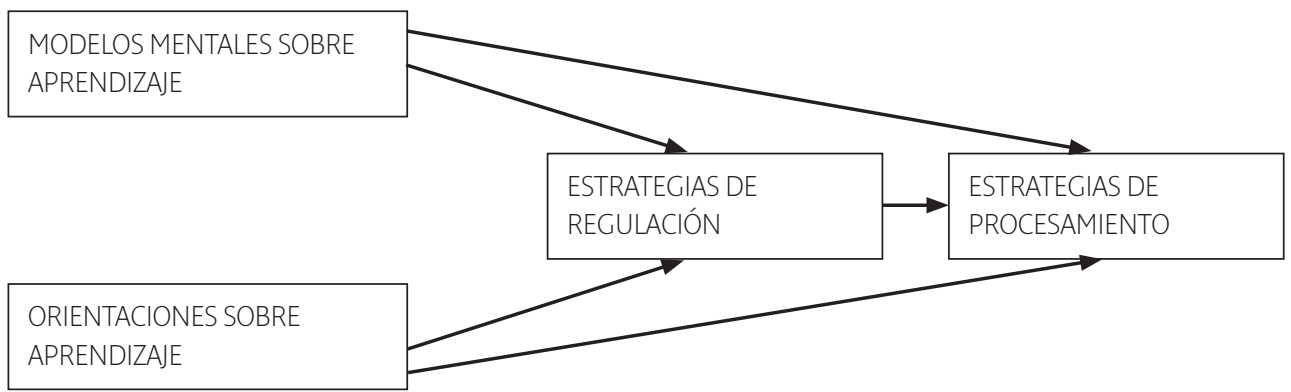

Fuente: Vermunt, 2005

Como ya hemos mencionado, un aspecto interesante del concepto de patrón es que es susceptible de cambiar según cambien también las circunstancias de la situación de aprendizaje (Donche y Van Petegem, 2009; Vermunt y Endedijk, 2011). A nuestro juicio, la maleabilidad en las formas de aprender abre la puerta a intervenciones educativas para favorecer patrones de aprendizaje probadamente eficaces para ciertos tipos de conocimiento o determinados contextos de interacción.

Vermunt (2005) propone cuatro patrones de aprendizaje básicos, a saber:

1.Patrón dirigido al significado (MD Meaning Directed). Este patrón describe aquellos estudiantes genuinamente interesados en el contenido de sus estudios, capaces de aprender de manera personal, autorregulada y responsable, y poseedores de habilidades para aproximarse al conocimiento de manera elaborada, profunda y crítica.

2.Patrón dirigido a la aplicación (AD Application Directed). Este patrón corresponde a aquellos estudiantes que privilegian la aplicación del conocimiento 
y asumen el aprendizaje como una actividad útil para el logro de objetivos personales ulteriores.

3.Patrón dirigido a la reproducción (RD Reproduction Directed). Este patrón se aplica a aquellos estudiantes cuyo objetivo principal es acumular conocimiento y reproducirlo durante las evaluaciones, para así aprobar los cursos y obtener un título profesional.

4.Patrón no dirigido (UD Undirected). Este patrón se atribuye a aquellos estudiantes que no tienen una razón clara de por qué están estudiando y pareciera que no saben en qué consiste aprender.

Dependiendo del contexto y de los propósitos de formación, al menos dos de los cuatro patrones básicos arriba descritos podrían ser útiles para el éxito académico tanto en estudiantes universitarios como en profesores en formación. En efecto, los patrones MD y AD favorecerían las condiciones para un proceso formativo hacia la excelencia (v.g. de la Barrera, Donolo y Rinaudo, 2010). Habría, por otra parte, un patrón indeseable a todas luces -el UD- y otro altamente deseable para una gran cantidad de propósitos de formación -el MD-(Vermunt y Donche, 2017).

Habida cuenta de lo anterior, resulta de gran utilidad pedagógica poder identificar el patrón que cada estudiante exhibe frente a un proceso de aprendizaje específico. Saber cómo aprenden sus estudiantes permitiría al profesor una planeación pedagógica que aproveche las fortalezas observadas y, al tiempo, intente cambiar patrones indeseables hacia enfoques más constructivos (v.g. Ruiz-Bueno y García Orriols, 2019).

Si bien se observa alguna actividad investigativa sobre patrones de aprendizaje en Hispanoamérica, como Argentina y México (de la Barrera et al., 2010; 2019; Gaeta González et al., 2020; García-Ravidá y González Velásquez, 2019; González y Difabio de Anglat, 2019) los estudios sobre este tema con población estudiantil colombiana están apenas comenzando. Al respecto, se destaca el trabajo de Martínez-Fernández y Vermunt (2015) sobre patrones de aprendizaje en estudiantes latinoamericanos, que incluye una pequeña muestra de estudiantes colombianos, y el de Martínez-Fernández, García-Ravidá y Mumbardó-Adam (2019), con 115 estudiantes que, precisamente, se encuentran en el primer año de su proceso de formación para ser profesores, en una institución de educación superior colombiana. En ambos casos, el interés investigativo apuntaba a la exploración de las formas en que los diferentes elementos del patrón de aprendizaje se agrupaban en esta población estudiantil y su relación con indicadores de rendimiento académico; por lo que no centraban su atención en la evolución de los patrones a lo largo de la carrera. 


\section{PATRONES DE APRENDIZAJE DE DOCENTES EN FORMACIÓN}

En líneas generales, el campo de investigación sobre patrones de aprendizaje ha puesto considerable atención sobre estudiantes que se forman para ser docentes. $\mathrm{Al}$ respecto, Donche y Van Petegem (2009) reportan un estudio que buscaba identificar en qué medida los componentes y los patrones de aprendizaje están sujetos a cambio durante un periodo de tiempo mayor a 12 meses. El estudio tuvo lugar con estudiantes belgas que se formaban para ser profesores, en un programa de 3 años de duración. En una primera fase, 646 estudiantes del primer año y 350 del tercer año respondieron el cuestionario ILS. En la segunda fase, 236 estudiantes de la misma universidad respondieron este mismo cuestionario dos veces, una al inicio del primer año y otra vez al final del tercer año de estudios.

En la primera parte del estudio se identificaron tres patrones de aprendizaje entre los estudiantes: un patrón que combinaba las tendencias RD y UD, un patrón claramente MD, y otro patrón denominado "flexible", caracterizado por una visión constructiva y aplicada del aprendizaje, acompañada del uso de estrategias tanto de autorregulación como de regulación externa y del manejo de estrategias de procesamiento profundas y concretas. Mientras los patrones MD y flexible se hacían más frecuentes durante el tercer año de estudios, el patrón RD/UD iba disminuyendo. Ahora bien, en lo que respecta al cambio de patrón en los estudiantes, aquellos que presentaban un patrón RD/UD en el primer año lo cambiaron en el tercero con un aumento de rasgos propios del patrón $\mathrm{MD}$, tales como el procesamiento concreto y las estrategias de autorregulación. Por su parte, los estudiantes que exhibían un patrón flexible durante el primer año mostraron una tendencia hacia el patrón MD en el tercero, en rasgos como el manejo de un procesamiento profundo y autorregulado, al tiempo que se disminuían características como la de concebir el aprendizaje como memorización de información. Finalmente, los estudiantes catalogados como MD en el primer año afianzaron este patrón aún más, con un repertorio amplio y flexible de estrategias de aprendizaje que incluye tanto procesos propios del patrón $\mathrm{MD}$ como de los patrones $\mathrm{AD}$ y $\mathrm{RD}$.

Por su parte, en el trabajo de Martínez-Fernández et al. (2019), los autores identificaron cuatro patrones presentes entre los futuros maestros: un patrón MD acompañado de regulación externa; un patrón identificado como "pasivo idealista" (PI), caracterizado por agrupar todas las concepciones de aprendizaje sin conexión con aspectos activos del proceso; un patrón "pasivo motivado" (PM), que agrupa todas las orientaciones hacia el aprendizaje y, finalmente, un patrón RD, acompañado de ausencia de regulación. Los autores explican la presencia de estos últimos tres patrones no presentes en el modelo inicial haciendo referencia al periodo de 
transición en el que los estudiantes se encontraban (terminando sus estudios teóricos e iniciando prácticas docentes). Si bien estos hallazgos son un antecedente importante para el presente estudio, deben tomarse con precaución dado el procedimiento utilizado para determinar los patrones, que se limita a un análisis factorial exploratorio sobre las diferentes escalas del instrumento, sin avanzar en la agrupación de los individuos por medio de un análisis de conglomerados. Este procedimiento podría ser considerado incompleto para la identificación de los patrones de aprendizaje de los individuos (Donche, Coertjens y Van Petegem, 2010; Donche y Van Petegem, 2009).

\section{Metodología}

\section{Tipo de estudio}

Se llevó a cabo un estudio descriptivo-observacional, transversal, en el que se pretende caracterizar los patrones de aprendizaje de una muestra aleatoria de la población estudiantil de una universidad pública dedicada a la formación de maestros en Colombia, en tres momentos de su formación: (1) al inicio, (2) en el intermedio y (3) al final de su carrera.

\section{Participantes}

Participaron en el estudio 1.016 estudiantes profesores en formación de cuatro diferentes Facultades de la universidad pedagógica mencionada. Esta universidad acoge población de estratos socioeconómicos medio bajos o bajos. La selección de la muestra se hizo de manera aleatoria, estratificada por momento de la carrera, bietápica y por conglomerados completos conformados por cursos académicos vigentes en el semestre.

Se buscó contar con estudiantes en tres momentos de sus carreras: al inicio (de $1^{\circ}$ a $2^{\circ}$ semestre), en un momento intermedio $\left(5^{\circ}\right.$ a $6^{\circ}$ semestre) y al final (entre los semestres $9^{\circ}$ y $10^{\circ}$ ). Los porcentajes de estudiantes renuentes a participar en el estudio fueron de menos del 1\%. En total, la muestra representa el 25,3\% del total de estudiantes de los semestres observados. La Tabla 1 presenta la muestra final efectiva, discriminándola por Facultad ${ }^{1}$ y momento de la carrera.

1. "Facultad" se refiere a una organización académico-administrativa de la universidad, que se diferencia según las áreas disciplinares específicas sobre las cuales el futuro maestro está siendo formado. 
PATRONES DE APRENDIZAJE DEL PROFESORADO COLOMBIANO EN FORMACIÓN

Tabla 1. Número de estudiantes de la muestra por Facultad y momento de la carrera

\begin{tabular}{clcccc}
\hline \multicolumn{5}{c}{ MOMENTO EN LA CARRERA } \\
\hline Facultad & INICIAL & INTERMEDIO & FINAL & TOTAL \\
\hline & Educación Física & 219 & 157 & 106 & 482 \\
\hline & Ciencia y Tecnología & 118 & 74 & 48 & 240 \\
\hline & Artes y Humanidades & 106 & 107 & 81 & 294 \\
\hline & Total & 443 & 338 & 235 & 1016 \\
\hline
\end{tabular}

Fuente: elaboración propia.

\section{Instrumento}

Inventory of Learning Styles (ILS). El ILS es un cuestionario de auto-reporte compuesto por 120 ítems y desarrollado por J. Vermunt en 1998. Permite la identificación de veinte escalas que se distribuyen en las diferentes dimensiones de los patrones de aprendizaje. La Tabla 2 muestra la distribución de las escalas del cuestionario ILS según el patrón de aprendizaje.

\section{Tabla 2. Escalas del ILS}

\begin{tabular}{lllll} 
DIMENSIÓN & $\begin{array}{l}\text { ORIENTADO AL } \\
\text { SIGNIFICADO (MD) }\end{array}$ & $\begin{array}{l}\text { ORIENTADO A LA } \\
\text { APLICACIÓN (AD) }\end{array}$ & $\begin{array}{l}\text { ORIENTADO ALA } \\
\text { REPRODUCCIÓN (RD) }\end{array}$ & $\begin{array}{l}\text { NO ORIENTADO } \\
\text { (UD) }\end{array}$ \\
\hline Creencias & $\begin{array}{l}\text { Construcción de } \\
\text { conocimiento }\end{array}$ & Uso del conocimiento & $\begin{array}{l}\text { Incremento del } \\
\text { conocimiento }\end{array}$ & $\begin{array}{l}\text { Cooperación. } \\
\text { Educación estimulante }\end{array}$ \\
\hline Orientaciones & Interés personal & Vocación. & Certificado. & Ambivalente \\
& & & Autoprueba. & \\
\hline Regulación & $\begin{array}{l}\text { Autorregulación } \\
\text { de procesos }\end{array}$ & & $\begin{array}{l}\text { Regulación externa } \\
\text { de proceso }\end{array}$ & Ausencia de regulación \\
& Autorregulación & & $\begin{array}{l}\text { Regulación externa } \\
\text { de resultados }\end{array}$ & \\
\hline Procesamiento resultados & Elaboración & Concreto & Memorización & \\
& Pensamiento crítico & & yensayo & \\
& & Análisis & \\
\hline
\end{tabular}

Fuente: Vanthournout, Donche, Gijbels y Van Petegem, 2014.

Este cuestionario ha mostrado adecuados niveles de validez y confiabilidad en poblaciones de diferente nivel educativo y procedencia geográfica y cultural (Boyle, 
Duffy y Dunleavy, 2003; Van der Veken, Valcke, Muijtjens, De Maeseneer y Derese, 2008). La versión en español del ILS fue traducida y validada por MartínezFernández, García-Orriols y Galera-Bassachs (2017) con muestras de población española y latinoamericana.

\section{Procedimiento}

A los estudiantes que asistían a los grupos seleccionados se le explicaron los propósitos del estudio. Una vez hecho esto, los asistentes firmaron el formato de consentimiento informado, un formato aprobado por el comité de ética de la universidad para este tipo de estudios, que involucra bajo riesgo para los participantes. El cuestionario ILS fue aplicado en los estudiantes que aceptaron participar en el estudio.

\section{Análisis}

Los datos fueron procesados bajo el paquete IBM-SPSS v. 25. En primer lugar, fueron examinados los niveles de confiabilidad obtenidos a través de los valores alfa de Cronbach para cada una de las subescalas que compone el instrumento.

Posteriormente, los puntajes de las subescalas fueron incluidos en un análisis de componentes principales, con rotación Varimax, para la identificación de la estructura interna de relaciones entre las diferentes subescalas y la obtención de puntajes factoriales ortogonales, que pudieran prestarse para ser los insumos para un análisis de conglomerados.

A continuación, se realizó un análisis de conglomerados sobre los puntajes factoriales obtenidos en el análisis anterior. Este fue realizado siguiendo un algoritmo bietápico, en el cual, primero se examinan las mejores formas de agrupar a los individuos en categorías claras y consistentes, y finalmente se asigna a cada individuo a una de las categorías. Estas categorías representan los patrones de aprendizaje encontrados en la presente muestra.

Finalmente, se examinaron las relaciones entre estos patrones y la pertenencia a las diferentes Facultades y momentos de la carrera. Para ello, se utilizaron pruebas de independencia Chi cuadrado de Pearson, con análisis de residuos estandarizados, para detectar casillas con valores inesperadamente altos, o bajos, en el cruce de las variables. 


\section{Alcances y limitaciones}

Los resultados pueden constituir una descripción muy precisa de un amplio conjunto de estudiantes de carreras de educación, que podría ser generalizable a estudiantes de las mismas carreras en otras universidades colombianas y latinoamericanas.

Debe anotarse una importante limitación de los resultados. En relación con el momento de la carrera, este es un estudio de tres paneles, más que un estudio longitudinal. Aunque puede simular estudios de tipo longitudinal, en sentido estricto no lo son, por lo que se recomienda precaución en la interpretación de esta sección de los resultados.

\section{RESUlTADOS}

\section{Confiabilidad de las escalas del ILS}

La Tabla 3 muestra los valores del alfa de Cronbach obtenidos en la presente aplicación del instrumento ILP para cada una de sus veinte escalas. Como se observa, en general, los valores de la confiabilidad tienden a ser altos.

Tabla 3. Valores del alfa de Cronbach en las escalas simples, agrupadas por dimensión y por patrón

\begin{tabular}{|c|c|c|c|c|}
\hline PATRÓN & DIMENSIÓN & NOMBRE DE LA ESCALA & ALFA & ITEMS \\
\hline \multirow[t]{6}{*}{ MD } & Creencias & Construcc. del conocimiento & ,769 & 9 \\
\hline & Motivación & Intereses personales &, 500 & 5 \\
\hline & \multirow[t]{2}{*}{ Regulación } & Autorreg. del contenido & ,688 & 4 \\
\hline & & Autorregulación del proceso & ,711 & 7 \\
\hline & \multirow[t]{2}{*}{ Procesamiento } & Procesamiento crítico & ,747 & 7 \\
\hline & & Establ. relaciones y estruct. & ,668 & 4 \\
\hline \multirow[t]{3}{*}{$A D$} & Creencias & Uso del conocimiento & ,693 & 6 \\
\hline & Motivación & Vocación & ,637 & 5 \\
\hline & Procesamiento & Procesamiento concreto &, 624 & 5 \\
\hline \multirow[t]{3}{*}{$\mathrm{RD}$} & Creencias & Obtención del conocimiento &, 756 & 9 \\
\hline & \multirow[t]{2}{*}{ Motivación } & Orientación al certificado &, 641 & 5 \\
\hline & & Dirigido a la autoprueba & ,737 & 5 \\
\hline
\end{tabular}


Tabla 3. Valores del alfa de Cronbach en las escalas simples, agrupadas por dimensión y por patrón

\begin{tabular}{lllrl} 
PATRÓN & DIMENSIÓN & NOMBRE DE LA ESCALA & ALFA & ITEMS \\
\hline RD & Regulación & Reg. externa del proceso &, 542 & 6 \\
\cline { 3 - 4 } & & Reg. externa de resultados &, 559 & 5 \\
\cline { 3 - 4 } & Procesamiento & Memorización y recup. &, 750 & 5 \\
\cline { 3 - 4 } & Creencias & Análisis &, 703 & 6 \\
\hline UD & Cooperación &, 801 & 8 \\
\cline { 3 - 5 } & Motivación & Ampectativa de estimulación &, 859 & 8 \\
\cline { 2 - 4 } & Regulación & Ausencia de regulación &, 685 & 5 \\
\hline
\end{tabular}

Fuente: elaboración propia.

Los valores más bajos de confiabilidad se presentan en la escala de intereses personales $(0,500)$ y en las escalas de regulación externa de proceso y de contenido $(, 542$ y ,559, respectivamente). Estos resultados son consistentes con otras aplicaciones del instrumento en el contexto latinoamericano, que muestran niveles de confiabilidad relativamente bajos para estas escalas específicas (Martínez-Fernández y Vermunt, 2015).

\section{Relaciones entre las escalas del ILS}

El segundo paso fue la realización de un análisis de componentes principales que permitiera examinar las relaciones entre las escalas y generar puntajes que pudieran ser insumos adecuados a un posterior análisis de conglomerados.

Los análisis preliminares mostraron la pertinencia de realizar el análisis de componentes principales sobre los datos; así lo indicaron el cálculo de la medida de Kaiser-Meyer-Olkin de adecuación muestral $\mathrm{KMO}=, 866$ y la prueba de esfericidad de Barlett $\chi^{2}(190)=7491,08 p<, 001$.

Los resultados del análisis de componentes principales, con rotación ortogonal Varimax, muestran la pertinencia de considerar cuatro factores. La Tabla 4 muestra la matriz de cargas rotadas con autovalores en cada factor y porcentajes de varianza explicados. En total, el modelo alcanza a explicar el 57,16\% de la varianza total. 
Tabla 4. Cargas rotadas y autovalores en el Análisis de Componentes Principales

\begin{tabular}{|c|c|c|c|c|}
\hline & 1 & 2 & 3 & 4 \\
\hline MD-PR Procesamiento crítico & 0,82 & & & \\
\hline MD-PR Establecer relaciones y estructuras & 0,80 & & & \\
\hline MD-RE Autorregulación del proceso & 0,79 & & & \\
\hline RD-PR Análisis & 0,74 & & 0,41 & \\
\hline MD-RE Autorregulación del contenido & 0,74 & & & \\
\hline AD-PR Procesamiento concreto & 0,68 & & & \\
\hline AD-CR Uso del conocimiento & & 0,74 & & \\
\hline MD-CR Construcción de conocimiento & 0,33 & 0,67 & & \\
\hline MD-MO Intereses personales & & 0,65 & & \\
\hline AD-MO Vocación & & 0,64 & & \\
\hline RD-MO Dirigido a la autoprueba & & 0,63 & & \\
\hline UD-CR Expectativa de estimulación & & 0,58 & & 0,35 \\
\hline UD-CR Cooperación & & 0,50 & & \\
\hline RD-RE Regulación externa del proceso & 0,31 & & 0,73 & \\
\hline RD-RE Regulación externa de resultados & & & 0,69 & \\
\hline RD-PR Memorización y recuperación & & & 0,64 & \\
\hline RD-MO Orientación al certificado & & 0,31 & 0,61 & \\
\hline RD-CR Obtención del conocimiento & & 0,51 & 0,58 & \\
\hline UD-MO Ambivalente & & & & 0,81 \\
\hline UD-RE Ausencia de regulación & & & & 0,68 \\
\hline Autovalor & 5,28 & 3,17 & 1,81 & 1,18 \\
\hline \% de varianza explicado & 20,17 & 16,59 & 12,93 & 7,48 \\
\hline \% de varianza acumulado (desp. rotación) & 20,17 & 36,75 & 49,69 & 57,16 \\
\hline
\end{tabular}

Nota: CR: Creencias, MO: Motivación, RE: Regulación, PR: Procesamiento. 
Las cargas factoriales se presentan ordenadas de forma descendente por factor. Se suprimen cargas factoriales $<, 30$ y se resaltan en negrita las mayores cargas de cada fila.

En el primer factor aparecen agrupadas seis escalas que tienen en común ser dimensiones de regulación o procesamiento de los patrones MD y AD. Podría considerarse que la escala de análisis es una excepción a esta regla puesto que el instrumento original la identificó como una dimensión de procesamiento del patrón RD. Esta agrupación de escalas ya había sido identificada antes y había sido rotulada como de escalas $\mathrm{MD}-\mathrm{AD}$ activas, en tanto que agrupa las dimensiones activas (de regulación y procesamiento) de estos dos patrones (Martínez-Fernández y Vermunt, 2015).

En el segundo factor aparecen siete escalas, que tienen en común ser las escalas de creencias y motivación de todos los patrones definidos por Vermunt (MD, $\mathrm{AD}, \mathrm{RD}$ y UD). Solo tres, del total de las diez escalas de creencias y motivación, no quedan acá ubicadas: dos escalas del patrón RD (orientación al certificado y obtención de conocimiento) y una escala del patrón UD (motivación ambivalente). En cada una de estas excepciones las escalas quedarán con las otras de su patrón correspondiente. Llamaremos a este factor el de escalas epistémicas (en tanto que agrupa, básicamente, creencias).

En el tercer factor aparecen cinco escalas, todas características del patrón RD. Las únicas escalas del patrón RD que no quedan en este factor son (1) la escala de análisis, que ya comentamos, y que no pertenece propiamente a este patrón, y (2) la escala de "dirigido a la autoprueba" (RD-MO), que se agrupa con otras creencias y deseos en el factor de escalas epistémicas. En gracia a su composición, denominaremos a este factor como el de escalas RD.

Finalmente, en el cuarto factor aparecen las dimensiones más características del patrón UD: la de motivación (ambivalente) y la de ausencia de regulación. Estas han sido las escalas más características de este patrón no dirigido. Vale la pena notar en este factor una cierta carga factorial apreciable de la escala de expectativa de estimulación (UD-CR) que es consistente con el patrón UD. Por su composición, llamaremos a este factor como el de escalas UD.

Para terminar con la descripción de la matriz de cargas factoriales, es interesante notar cargas, marginales pero observables, de las escalas de creencias y de motivación del patrón RD en el factor 2, que resultan perfectamente consistentes con la naturaleza de este factor de escalas epistémicas. De igual forma, la escala de análisis (RD-PR) muestra también una carga apreciable en el factor 3 (RD), lo que resulta consistente con la inicial definición de Vermunt para esta escala. 
Patrones de aprendizaje a partir del análisis de conglomerados

A partir de los puntajes factoriales obtenidos en el análisis anterior, se utilizó el algoritmo bietápico para la realización de una clasificación de individuos que permitiera identificar patrones de aprendizaje en la muestra. El algoritmo recomienda una clasificación en cuatro conglomerados que contienen, el primero, 271 individuos (26,7\%), el segundo 267 (26,3\%), 190 (18,7\%) el tercero y 288 (28,3\%) el cuarto. La Figura 2 presenta las medias de cada factor para cada conglomerado.

Figura 2. Medias de cada factor en los conglomerados

1,50

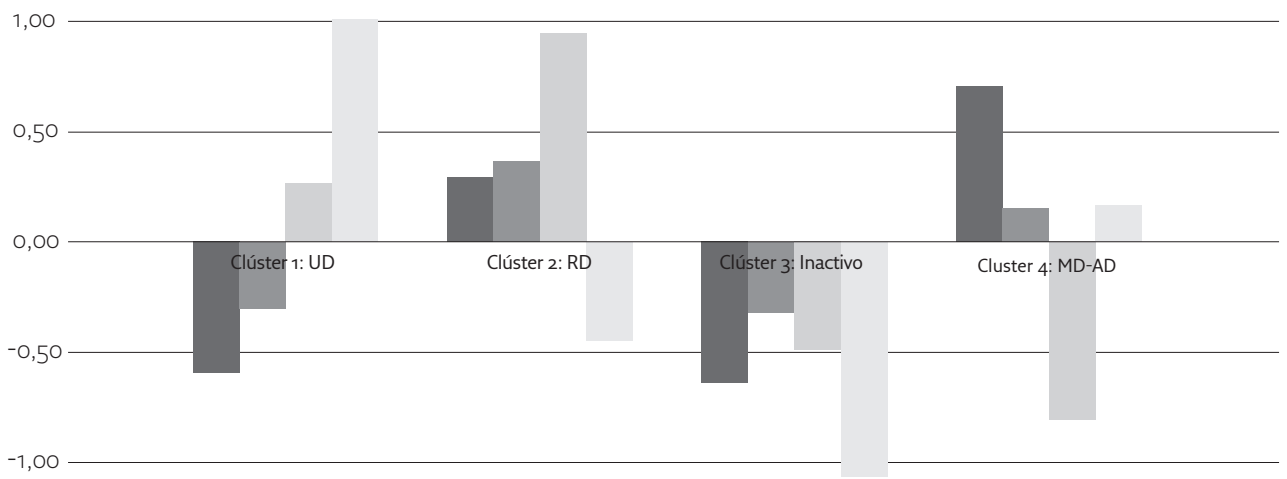

MDAD Activas $\square$ Epistémicas $\square$ RD $\square$ UD

Fuente: elaboración propia.

El primer conglomerado (primer grupo de barras en la gráfica) muestra puntajes muy bajos en el primer factor (MD-AD), puntajes medios en los factores 2 y 3 y puntajes muy altos en el cuarto (UD). Por estas características, diríamos que este conglomerado está compuesto por individuos pertenecientes al patrón no dirigido (UD).

El segundo de los conglomerados (segundo grupo de barras en la gráfica) muestra puntajes muy altos en el tercer factor (RD), medios en los factores 1 y 2 y muy bajos en el cuarto factor (UD). Así, esta categoría está compuesta por individuos orientados al patrón RD. 
El tercer conglomerado (tercer grupo de barras en la gráfica) muestra puntajes muy bajos en todos los factores, y especialmente bajos en el cuarto factor (UD). Aunque la ausencia de puntajes altos en algún factor dificulta, inicialmente, su identificación con un patrón de aprendizaje, este tipo de sesgo de respuesta puede estar identificando un grupo que no está siendo caracterizado positivamente por el instrumento marcado por su escepticismo. Vermetten, Vermunt y Lodewijks (2002) habían reportado el hallazgo de un grupo similar en estudiantes universitarios holandeses, a los que llamaron "aprendices inactivos" (inactive learners), caracterizado por bajos niveles de respuesta en todas las actividades de aprendizaje. Adoptaremos esta misma denominación para este grupo.

Por último, el cuarto de los conglomerados muestra muy altos puntajes en el primer factor (MD-AD), puntajes medios en los factores 2 y 4 y puntajes muy bajos en el tercer factor (RD). Por estas características podemos decir que los individuos en este conglomerado presentan las acciones que caracterizan los patrones MD y $\mathrm{AD}$ y que, al tiempo, los distancian del patrón orientado a la reproducción. Los individuos de este conglomerado son aquellos que, en sus comportamientos, resultan ser los más cercanos a los patrones MD y $\mathrm{AD}$.

\section{Relaciones con el momento de la carrera}

En relación con el momento de la carrera, parece haber diferencias significativas en las proporciones de los grupos de patrones de aprendizaje. Tal y como se observa en la Figura 3, la proporción de población con los patrones UD y RD muestra ser bastante abultada al inicio de la formación, disminuyendo de forma apreciable en el momento intermedio y llegando a su mínimo en el momento final. Al respecto del tercer y cuarto conglomerado, que hemos denominado aprendices "inactivos" y $\mathrm{MD}-\mathrm{AD}$, respectivamente, la tendencia es la contraria: en el momento inicial muestran proporciones relativamente pequeñas que van creciendo en los momentos posteriores y llegan a su máximo en el momento final, hecho particularmente visible en los individuos del patrón MD-AD.

El análisis de la independencia estadística entre estos dos aspectos se hizo a través de una prueba Chi cuadrado de Pearson. Los resultados mostraron una asociación muy significativa entre el patrón, entendido como la pertenencia al conglomerado, y el momento de la carrera, con un tamaño del efecto, medido a través del coeficiente $V$ de Cramer, que puede considerarse entre mediano y grande (Kim, 2017) $\chi^{2}(6)=40,63 p<, 001 V=0,14$. 
Figura 3. Proporción de patrones por momento de la carrera

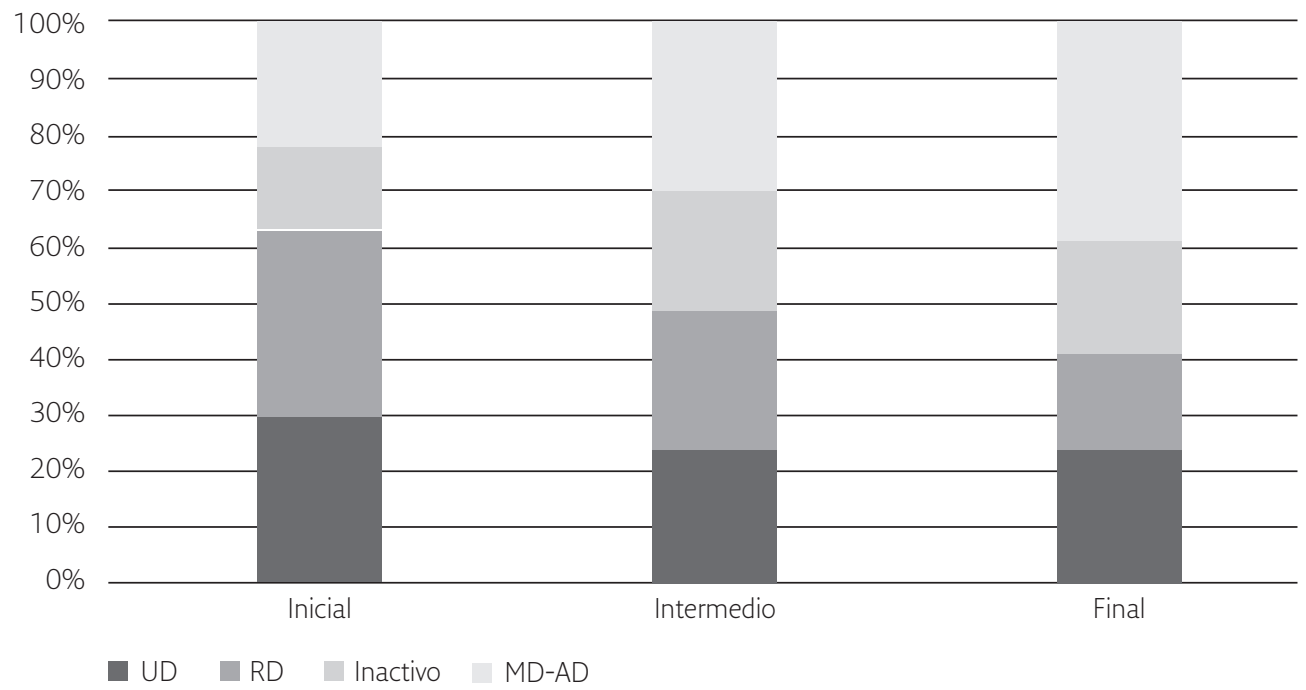

Fuente: elaboración propia.

Para el examen de las diferencias específicas entre las proporciones esperadas por cada casilla de la tabla de cruce, que sería equivalente a la realización de pruebas post hoc, se examinan a los residuos estandarizados corregidos de la tabla de cruce de variables. El criterio es simple: si en una casilla, el residuo estandarizado es mayor a 1,96 en valor absoluto, decimos que hay una diferencia significativa en ese valor de ese grupo específico; si es negativo, la casilla muestra un valor significativamente menor, si es positivo será significativamente mayor. La Tabla 5 muestra el cruce entre el patrón de aprendizaje y el momento de la carrera, con los residuos estandarizados. 
HEDERICH-MARTÍNEZ / CAMARGO-URIBE / HERNÁNDEZ-VALBUENA

Tabla 5. Tabla de cruce (con residuos estandarizados) entre patrón de aprendizaje y momento de la carrera

\begin{tabular}{llcccc} 
& & \multicolumn{3}{c}{ MOMENTO EN LA CARRERA } & TOTAL \\
\cline { 2 - 5 } & & INICIAL & INTERMEDIO & FINAL \\
\hline \multirow{3}{*}{$\begin{array}{l}\text { Patrón de } \\
\text { aprendizaje }\end{array}$} & $132\left(2,0^{*}\right)$ & $81(-1,4)$ & $58(-0,8)$ & 271 \\
\cline { 2 - 5 } & C2: RD & $146\left(4,3^{*}\right)$ & $83(-0,9)$ & $38\left(-4,0^{*}\right)$ & 267 \\
\cline { 2 - 5 } & C3: Inactivo & $67\left(-2,6^{*}\right)$ & $75(2,0 *)$ & $48(0,8)$ & 190 \\
\cline { 2 - 5 } & C4: MD AD & $98\left(-3,9^{*}\right)$ & $99(0,5)$ & $91\left(4,0^{*}\right)$ & 288 \\
\hline Total & 443 & 338 & 235 & 1.016 \\
\hline
\end{tabular}

Valores (con residuos estandarizados corregidos entre paréntesis)

*: $p<, 05$

Como se observa, en el momento inicial de la carrera los estudiantes son predominantemente de los patrones RD y UD y muy especialmente del primero, mientras que los patrones de aprendices inactivos y $\mathrm{MD}-\mathrm{AD}$ aparecen muy poco representados. Para el momento intermedio de la carrera se observa un incremento importante en el grupo de aprendices inactivos, mientras que los otros tres conglomerados se mantienen sin variaciones mayores. Ya para el final de la carrera lo observado al inicio se invierte: los estudiantes en ese momento muestran un patrón predominante de las formas $\mathrm{MD}$ y $\mathrm{AD}$, mientras que la proporción de estudiantes del patrón $\mathrm{RD}$ es significativamente menor.

\section{DisCUSIÓN Y CONCLUSIONES}

Los resultados ofrecen un panorama general de la población estudiantil de una universidad pública que forma maestros respecto de sus patrones de aprendizaje y de sus transformaciones a lo largo de la carrera.

Primero, deben comentarse las características técnicas del instrumento ILS a partir de lo encontrado en la muestra. En general, la confiabilidad de las diferentes subescalas del instrumento resulta satisfactoria, si bien debe notarse un valor particularmente bajo para la escala de intereses personales, que representa la dimensión motivacional del patrón MD. En otros estudios, esta escala ha mostrado niveles de consistencia bastante menores que las otras escalas motivacionales y del patrón MD (Martínez-Fernández y Vermunt, 2015; Martínez-Fernández et al., 2019).

Otro tanto ocurre con la escala de análisis (RD-PR), originalmente asumida como parte del componente de procesamiento del patrón RD. En otras oportuni- 
dades esta escala ha mostrado asociaciones consistentes, no con las otras escalas del patrón $\mathrm{RD}$, sino con las escalas de regulación y procesamiento de los patrones MD y AD (Martínez-Fernández y Vermunt, 2015; Martínez-Fernández et al., 2019). Este hecho parece identificar al procesamiento analítico, más como característica del patrón $\mathrm{AD}$, que del RD.

En segundo lugar, debe comentarse la persistente asociación encontrada, en los patrones $\mathrm{MD}$ y $\mathrm{AD}$, entre las escalas de creencias y orientación, que les lleva a formar grupo aparte de las escalas de regulación y procesamiento. Esta parece ser una característica particular de los estudiantes de carreras de educación en Latinoamérica (Martínez-Fernández y Vermunt, 2015; Martínez-Fernández et al., 2019). La estructura de asociaciones podría sugerir un sesgo de deseabilidad social, que surge como resultado de la formación de los estudiantes en los temas relacionados con las teorías del aprendizaje mencionadas en el instrumento mismo. Esta separación tiene como resultado el hecho de que las dimensiones activas del patrón (regulación y procesamiento) resultan ser mejores indicadores de los patrones MD y $\mathrm{AD}$ que las dimensiones epistémicas (de creencias y orientaciones). Así, una buena parte de los futuros maestros parecen adoptar un discurso avanzado sobre el aprendizaje, en el que se incorporan elementos de la teoría constructivista, mientras continúan con acciones que no representan estas creencias (Hederich-Martínez y Camargo-Uribe, 2019).

Este resultado se opone a lo encontrado en algunos estudios previos, en los que los modelos mentales y las orientaciones de cada patrón se conjugan armónicamente con sus dimensiones regulatorias y estratégicas (Edelbring, 2012; Loyens, Rikers y Schmidt, 2008), pero es consistente con lo encontrado en otros trabajos en los que se constata esa disonancia (Martínez-Fernández y Vermunt, 2015).

Tercero, ya en relación con los patrones encontrados a partir del análisis de conglomerados, los resultados son prueba de la utilidad del modelo de Vermunt para caracterizar las formas de aprender en contextos universitarios colombianos, pero plantean algunas diferencias. Una de las más interesantes es la aparición de un patrón de estudiantes "inactivos", caracterizados por sus bajas respuestas en todas las escalas del instrumento. Este patrón ha aparecido en otras ocasiones (p.e. VegaRamírez, Hederich-Martínez y Vidaci, 2020; Vermetten, Vermunt y Lodewijks, 2002) y amerita una interpretación. De acuerdo con nuestra actual concepción al respecto, este patrón puede representar a un grupo que no está siendo adecuadamente representado por el instrumento. Muy posiblemente este patrón represente un estado de transición entre el patrón $\mathrm{RD}$, centrado en la acumulación de conocimiento, y los patrones $\mathrm{MD}$ y $\mathrm{AD}$, ligados a la construcción de significados. Obsérvese que este patrón parece incrementarse de forma notable en los momen- 
tos intermedios de la carrera, entre los patrones RD y UD propios del inicio, y el patrón MD-AD más presente hacia el final.

A pesar de que los resultados no surgen de un estudio longitudinal, sino de un estudio transversal de paneles, las estructuras de asociación entre el patrón de aprendizaje y el momento de la carrera son consistentes con lo encontrado en la investigación previa e indican, en términos generales, una transformación de los estudiantes hacia patrones de aprendizaje más evolucionados y complejos (Donche y Van Petegem, 2009). Las transformaciones observadas son las esperadas: a medida que se avanza en la carrera, las tendencias reproductivas y repetitivas se van abandonando para dar paso a una aproximación más profunda, reflexiva y crítica frente al conocimiento. La presencia de estudiantes inactivos a mitad de la carrera sugiere la presencia de un patrón intermedio en la evolución. Por otro lado, la presencia de un segmento importante de población, que supera el 20\%, con características del patrón UD es preocupante y requiere de más investigación.

Una alternativa de interpretación, no necesariamente contradictoria, surge de la consideración de que los estudiantes de semestres superiores son precisamente aquellos que han superado las pruebas y retos académicos que supone un proceso formativo. En este sentido, las diferencias observadas podrían explicarse en parte por la selección de aquellos estudiantes que presentan los patrones más elaborados desde el inicio, lo que incrementaría su probabilidad de permanencia. No podemos negar de plano esta interpretación, pero para poder valorarla en su justa medida, se requeriría otro tipo de diseño metodológico de carácter longitudinal. En futuras investigaciones habrá de explorarse esa posibilidad.

Fecha de recepción del original: 13 de octubre 2021

Fecha de aceptación de la versión definitiva: 1 de diciembre 2021

\section{REFERENCIAS}

Boyle, E. A., Duffy, T. y Dunleavy, K. (2003). Learning Styles and Academic Outcome: The validity and utility of Vermunt's Inventory of Learning Styles in a British higher education setting. British Fournal of Educational Psychology, 73, 267-290. https://doi.org/10.1348/00070990360626976

Camargo-Uribe, A. (2015). El estilo de enseñanza. Una mirada comunicativa y didáctica en el aula de ciencias naturales. Bogotá: Universidad Pedagógica Nacional.

de la Barrera, M. L., Donolo, D. y Rinaudo, M. C. (2010). Estilos de aprendizaje en alumnos universitarios: peculiaridades al momento de aprender. Revista Estilos de Aprendizaje, 3(6), 1-25. http://revistaestilosdeaprendizaje.com/article/view/912 
de la Barrera, M. L., Rigo, D. y Riccetti, A. (2019). La investigación en patrones de aprendizaje en Argentina: conformación de perfiles en estudiantes de Educación Superior. Revista Colombiana de Educación, 1(77). https://doi. org/10.17227/rce.num77-9521

Donche, V., Coertjens, L. y Van Petegem, P. (2010). Learning Pattern Development Throughout Higher Education: A longitudinal study. Learning and Individual Differences, 20, 256-259. https://doi.org/10.1016/j.lindif.2010.02.002

Donche, V. y Van Petegem, P. (2009). The Development of Learning Patterns of Student Teachers: A cross-sectional and longitudinal study. Higher Education, 57, 463-475. https://doi.org/10.1007/s10734-008-9156-y

Edelbring, S. (2012). Measuring Strategies for Learning Regulation in Medical Education: Scale reliability and dimensionality in a Swedish sample. $B M C$ Medical Education, 12, article number 76. https://doi.org/10.1186/1472-6920$12-76$

Finson, K. D., Thomas, J. E. y Pedersen, J. A. (2006). Comparing Science Teaching Styles to Students' Perceptions of Scientists. School Science and Mathematics, 106(1), 8-15. https://doi.org/10.1111/j.1949-8594.2006.tb18066.x

Gaeta González, M. L., Espinosa Jiménez, M., Reyes Vergara M. L., Gutiérrez Niebla, M. I., González Rabino, M. L., Benítez Ríos, Y. T. y García-Béjar, L. (2020). Perspectiva de futuro, patrones de aprendizaje y rendimiento académico en estudiantes universitarios mexicanos. Estudios sobre Educación, 39, 9-31. https://doi.org/10.15581/004.39.9-31

García-Ravidá, L. B. y González Velázquez, L. (2019). Patrones de aprendizaje en el contexto universitario mexicano: revisión sistemática del modelo y de sus publicaciones. Revista Colombiana de Educación, 1(77). https://doi.org/10.17227/ rce.num77-9538

González, M. L. y Difabio de Anglat, H. E. (2019). Vinculación entre patrones de aprendizaje y patrones temporales en estudiantes universitarios argentinos. Revista Colombiana de Educación, 1(77). https://doi.org/10.17227/rce.num779597

Hederich-Martínez, C. y Camargo-Uribe, A. (2019). Revisión crítica del modelo de patrones de aprendizaje de J. Vermunt. Revista Colombiana de Educación, 77, 344-368. https://doi.org/10.17227/rce.num77-9469

Loyens, S., Rikers, R. y Schmidt, H. (2008). Relationships between students' conceptions of constructivist learning and their regulation and processing strategies. Instructional Science, 36, 445-462. https://doi.org/10.1007/s11251-0089065-6

Martínez-Fernández, J. R., García-Orriols, J. y Galera-Bassachs, A. (2017). Patro- 
nes de aprendizaje en educación Primaria: identificación y acciones formativas inclusivas. Barcelona: Wolters Kluwer.

Martínez-Fernández, J. R., García-Ravidá, L. y Mumbardó Adam, C. (2019). Latin American undergraduates and learning patterns in the transition to higher education: an exploratory study in Colombia. Electronic Fournal of Research in Educational Psychology, 17(3), 561-588. https://doi.org/10.25115/ejrep. v17i49.2421

Martínez-Fernández, J. R. y Vermunt, J. (2015). A Cross-cultural Analysis of Patterns of Learning and Academic Performance of Spanish and Latin-American Undergraduates. Studies in Higher Education, 40(2), 278-295. https://doi.org/ 10.1080/03075079.2013.823934

Ruiz-Bueno, C. y García-Orriols, J. (2019). ¿Qué nos aporta el modelo de patrones de aprendizaje para el diseño de acciones formativas? Revista Colombiana de Educación, 1(77). https://doi.org/10.17227/rce.num77-9527

Saracho, O. (2003). Matching Teachers and Students Cognitive Styles. Early Child Development and Care, 173(2-3), 161-173. https://doi. org/10.1080/03004430303097

Vanthournout, G., Donche, V., Gijbels, D. y Van Petegem, P. (2014). (Dis)similarities in research on learning approaches and learning patterns. En G. Gijbels, V. Donche, J. Richardson y J. Vermunt (Eds.), Learning patterns in bigher education (pp. 9-32). Londres: Routledge.

Van der Veken, J., Valcke, M., Muijtjens, A., De Maeseneer, J. y Derese, A. (2008). The Potential of the Inventory of Learning Styles to Study Students' Learning Patterns in Three Types of Medical Curricula. Medical Teacher, 30, 863869. https://doi.org/10.1080/01421590802141167

Vega-Ramírez, L., Hederich-Martínez, C. y Vidaci, A. (2020). Identificación de los Patrones de aprendizaje: estudiantes de Ciencias de la Actividad Física y el Deporte y Maestro en Educación Primaria. En R. Roig-Vila (Ed.) La docencia en la Enseñanza Superior. Nuevas aportaciones desde la investigación e innovación educativas (pp. 454-460). Barcelona: Octaedro.

Vermetten, Y. J., Vermunt, J. D. y Lodewijks, H. G. (2002). Powerful learning environments? How university students differ in their response to instructional measures. Learning and Instruction, 12, 263-284. https://doi.org/10.1016/ S0959-4752(01)00013-5

Vermunt, J. D. (1998). The regulation of constructive learning processes. British Fournal of Educational Psychology, 68, 149-171. https://doi. org/10.1111/j.2044-8279.1998.tb01281.x

Vermunt, J. D. (2005). Relations between Student Learning Patterns and Perso- 
nal and Contextual Factors and Academic Performance. Higher Education, 49, 205-234. https://doi.org/10.1007/s10734-004-6664-2

Vermunt, J. D. (2007). The power of teaching-learning environments to influence student learning. British Fournal of Educational Psychology, Monograph Series II, 4, 73-90.

Vermunt, J. D. y Donche, V. (2017). A Learning Patterns Perspective on Student Learning in Higher Education: State of the art and moving forward. Educational Psychology Review, 29(2), 269-299. https://doi.org/10.1007/s10648-0179414-6

Vermunt, J. D. y Endedijk, M. D. (2011). Patterns in teacher learning in different phases of the professional career. Learning and Individual Differences, 21(3), 294-302. https://doi.org/10.1016/j.lindif.2010.11.019

Vermunt, J. D. y Verloop, N. (1999). Congruence and Friction between Learning and Teaching. Learning and Instruction, 9, 257-280. https://doi.org/10.1016/ S0959-4752(98)00028-0 
